\title{
Tocilizumab in Hospitalized Patients with COVID-19: A Meta Analysis of Randomized Controlled Trials
}

\author{
Vijairam Selvaraj ${ }^{1,2}$ (1) Mohammad Saud Khan ${ }^{1,2} \cdot$ Chirag Bavishi $^{2,3} \cdot$ Kwame Dapaah-Afriyie $^{1,2} \cdot$ Arkadiy Finn $^{1,2}$. \\ Amos Lal $^{4}$ - Eleftherios Mylonakis ${ }^{2,5}$
}

Received: 16 April 2021 / Accepted: 11 May 2021 / Published online: 29 May 2021

(c) The Author(s), under exclusive licence to Springer Science+Business Media, LLC, part of Springer Nature 2021

\begin{abstract}
Background To date, only dexamethasone has been shown to reduce mortality in coronavirus disease-19 (COVID-19) patients. Tocilizumab has been recently added to the treatment guidelines for hospitalized COVID-19 patients, but data remain conflicting.

Study Design and Methods Electronic databases such as MEDLINE, EMBASE, and Cochrane central were searched from March 1, 2020, until March 10, 2021, for randomized controlled trials evaluating the efficacy of tocilizumab in hospitalized COVID-19 patients. The outcomes assessed were all-cause mortality, mechanical ventilation, and time to discharge.

Results Nine studies (with 6490 patients) were included in the analysis. In total, 3358 patients received tocilizumab, and 3132 received standard care/placebo. Pooled analysis showed a significantly decreased risk of all-cause mortality (RR 0.89, $95 \%$ CI $0.80-0.98, p=0.02$ ) and progression to mechanical ventilation (RR $0.80,95 \%$ CI $0.71-0.89, p<0.0001$ ) in the tocilizumab arm compared to standard therapy or placebo. In addition, there was a trend towards improved median time to hospital discharge (RR 1.28, 95\% CI 1.12-1.45, $p=0.0002$ ).

Conclusions Tocilizumab therapy improves outcomes of mortality and need for mechanical ventilation, in hospitalized patients with COVID-19 infection compared with standard therapy or placebo. Our findings suggest the efficacy of tocilizumab therapy in hospitalized COVID-19 patients and strengthen the concept that tocilizumab is a promising therapeutic intervention to improve mortality and morbidity in COVID-19 patients.
\end{abstract}

Keywords COVID-19 $\cdot$ Tocilizumab $\cdot$ IL-6 $\cdot$ Mortality $\cdot$ Meta-analysis

$\begin{array}{ll}\text { Abbreviations } & \\ \text { BACC } & \begin{array}{l}\text { Boston Area COVID-19 } \\ \text { Consortium }\end{array} \\ \text { RCT-TCZ-COVID-19 } & \begin{array}{l}\text { Randomized controlled } \\ \text { trial-tocilizumab-COVID-19 }\end{array}\end{array}$

Vijairam Selvaraj

vijairam.selvaraj@lifespan.org

1 Division of Medicine, The Miriam Hospital, 164 Summit Ave, Providence, RI 02906, USA

2 Division of Medicine Aff2, Warren Alpert Medical School of Brown University, Providence, RI, USA

3 Division of Cardiology, Cardiovascular Institute, Rhode Island Hospital, Providence, RI, USA

4 Division of Pulmonary and Critical Care Medicine, Mayo Clinic, Rochester, MN, USA

5 Division of Infectious Diseases, Rhode Island Hospital, Providence, RI, USA
CORIMUNO-TOCI-1 Cohort multiple randomized controlled trials open-label of immune modulatory drugs and other treatments in COVID-19 patients

EMPACTA

Evaluating Minority Patients with Actemra

REMAP-CAP

Randomized, embedded, multifactorial adaptive platform trial for community-acquired pneumonia

RECOVERY Randomized evaluation of COVID-19 therapy

MEDLINE

Medical literature analysis and retrieval system online

EMBASE Excerpta Medica database 


\section{Introduction}

The coronavirus disease-19 (COVID-19) pandemic has resulted in hospitalization in many cases. In severe and critical cases of COVID-19, which occurs in $13.8 \%$ and $6.1 \%$ of the patient population, COVID-19-associated pneumonia can lead to acute respiratory distress syndrome (ARDS) and rapid deterioration, sometimes leading to invasive mechanical ventilation and death [1]. The clinical spectrum of COVID-19 continues to evolve along with the emergence of new severe acute respiratory syndromecoronavirus-2 (SARS-CoV2) variants.

The pathophysiology of COVID-19 involves an initial viremic phase where patients mostly have mild constitutional symptoms, followed by a pulmonary and then hyperinflammatory phase where patients have shortness of breath and hypoxemia [2]. The median time from onset of symptoms to hospital admission is five to seven days and nine to ten days for acute respiratory distress syndrome (ARDS) and hypoxic respiratory failure [3, 4]. The hyperinflammatory phase of COVID-19 is associated with elevated C-reactive protein (CRP) levels, ferritin, lactate dehydrogenase (LDH), and interleukin-6 (IL-6) and causes edema and inflammatory cell infiltration in the lungs [5, 6]. IL-6 appears to play a significant role in endothelial dysfunction and the development of vascular permeability and has been associated with the vascular dysfunction seen in severe disease [7]. This dysregulated and excess immune response plays an important role in the disease course of COVID-19 [8, 9]. Elevated levels of IL-6 have been associated with prolonged viral shedding, increased viremia, and progression to mechanical ventilation and death [10-13]. A meta-analysis of 6 studies revealed that mean IL-6 levels were 2.9-fold higher in patients with complicated COVID-19 than non-complicated disease [14].

Dexamethasone therapy has shown mortality benefit in patients of COVID-19 infection [15, 16]. Nevertheless, in some severely ill patients, dexamethasone therapy alone might not be sufficient to quell the cytokine storm in the hyperinflammatory phase. Tocilizumab is a recombinant monoclonal antibody indicated for treating giant cell arteritis, rheumatoid arthritis, and life-threatening cytokine release syndrome induced by chimeric antigen receptor T-cells [17-19]. Its mode of action is by inhibiting IL-6 signaling by binding soluble IL-6R and membrane IL-6R [20]. A recent study also showed there might be genetic variants in the interleukin-6 inflammatory pathway that may be associated with life-threatening disease in COVID19 patients [21]. It is reasonable to assume that early intervention with tocilizumab through IL-6 blockade could abrogate progression to hypoxemic respiratory failure and decreases the duration of supplemental oxygen use [22]. However, randomized controlled trials (RCTs) and systematic reviews evaluating the role of tocilizumab in COVID-19 patients have yielded disparate results [23-29].

We, therefore, conducted a meta-analysis of the RCTs to synthesize the current evidence on the efficacy of tocilizumab in hospitalized COVID-19 patients.

\section{Methods}

\section{Data Sources and Search Strategy}

This study was conducted according to the Cochrane Collaboration and the preferred reporting items for systematic reviews and meta-analyses (PRISMA) statement [30]. We conducted a systematic search in MEDLINE, EMBASE, Cochrane Central, and preprint databases to identify all relevant articles using the following search terms: "SARSCoV2" OR "COVID-19") AND ("tocilizumab" OR "IL6" OR "Anti-IL6"). Results were limited to humans and the English language. Databases were searched from March 1, 2020, to March 10, 2021, to identify all relevant RCTs including preprint, non-peer reviewed studies as well. Review articles, case reports, observational studies, opinion articles, letters, abstracts, conferences, brief reports, and non-English publications were excluded. The reference lists of the identified articles were also perused to find additional pertinent studies. All results were imported into EndNote $\times 8.2$ (Clarivate Analytics) and duplicate results were identified and removed.

\section{Study Selection and Eligibility}

Two independent reviewers (V.S. and M.S.K.) screened the retrieved papers based on the title and abstract. If the paper contained relevant data, the full paper was retrieved if it was not clear from the title and abstract. Any disagreements between the two reviewers were discussed with a third reviewer (K.D.A.) and resolved by consensus. A study was considered eligible for inclusion in the analysis if it was 1) randomized controlled trial 2) reported outcomes of interest in hospitalized COVID-19 patients with tocilizumab therapy compared to standard treatment or placebo. The outcomes assessed were all-cause mortality, progression to mechanical ventilation, and the median time to hospital discharge. If more than one study reported data from the same population, then the largest study was included.

\section{Data Extraction}

From the included studies, two reviewers (V.S. and M.S.K.) independently extracted the data. Extracted data included (1) 
study characteristics-design, site of study, dates of study, and type of randomization (2) details of the study population and the interventions utilized, including demographics of participants in both intervention and control arms, presence of comorbidities, concomitant treatment, CRP levels (3) primary outcome, and follow-up. In the REMAP-CAP trial, data from only the tocilizumab arm were included. Data from the sarilumab arm of the trial were excluded from the meta-analysis [31].

\section{Risk of Bias Assessment}

Two authors (V.S. and M.S.K.) reviewed each selected trial for quality assessment, including the risk of bias using the Cochrane criteria for systematic review of interventions [32]. This methodology explores the adequacy of sequestration, allocation sequence concealment, blinding of participants and study personnel, blinding for outcome assessment, incomplete outcome or selective outcome reporting, and another potential bias. Any disagreement between the authors was resolved with mutual agreement after discussion.

\section{Data Synthesis and Statistical Analysis}

Outcomes were used in the meta-analysis only if at least three studies reported usable data. The Mantel-Haenszel method for dichotomous data was used to calculate aggregated risk ratios (RRs) with corresponding $95 \%$ confidence intervals (CIs). A 2-tailed alpha level of 0.05 was set as the threshold for statistical significance. The $I^{2}$ statistic was used to assess unexplained statistical heterogeneity among studies. The meta-analysis was performed with a random-effects model. Subgroup analysis was done to evaluate the effect of disease severity, dexamethasone use, and sample size on all-cause mortality. Statistical analysis was performed using Review Manager, version 5.3 (Copenhagen: The Nordic Cochrane Centre, The Cochrane Collaboration, 2014).

\section{Results}

\section{Search Results and Characteristics of Included Trials}

Figure 1 shows the PRISMA flow chart summarizing the search strategy. The literature search identified 38 full-text articles, of which nine RCTs were eligible for inclusion in this study after full read [24-29, 31, 33]. A total of 6490 patients were included, of which 3358 patients received tocilizumab, and 3132 received standard care/placebo. Baseline characteristics were similar across the intervention and standard care or placebo groups. Detailed characteristics of the studies are described in Table 1.
All trials included in the analysis studied hospitalized patients with COVID-19 and were multicenter in design. One study was conducted in Italy, one in Brazil, one in India, one in France, one in the United Kingdom, and one in the United States of America (USA). Three international trials were conducted across multiple countries in Europe, Mexico, Kenya, South Africa, Peru, and Brazil [25, 26, 31]. Six trials had open-label design and three were double-blinded. Detailed study designs and study criteria are described in Table 2.

\section{Risk of Bias Assessment}

The risk of bias assessment for the included trials is presented in Fig. 2. All trials were reported using random sequence generation. Concealment of allocation was not mentioned in six trials and therefore risk of allocation concealment remains high in these trials [27-29, 31, 33, 34]. Also, six trials had open-label study design [27-29, 31, 33, 34]. The risk of performance and selection bias was high in these trials as participants and personnel were not blinded to the assigned treatment. Bias due to selective reporting was deemed high in one study because it was a preliminary report and data were not presented for some outcomes [31]. Risk of attrition bias was deemed high in one trial due to incomplete outcome [29]. In the rest of the studies, risk of attrition bias was low [24-28, 31, 33, 34].

\section{Assessment of Outcomes}

\section{All-Cause Mortality}

All nine RCTs reported the outcome of all-cause mortality [24-29, 31, 33, 34]. A total of 846 deaths out of 3358 participants were reported in the tocilizumab arm compared to 943 deaths out of 3132 participants in the standard care or placebo arm. Pooled analysis showed a significant reduction in all-cause mortality with tocilizumab therapy than standard therapy or placebo (RR $0.89,95 \%$ CI $0.80-0.98, p=0.02$ ) (Fig. 3A).

\section{Progression to Mechanical Ventilation}

Eight RCTs reported the outcome of progression to mechanical ventilation [24, 25, 27-29, 31, 33, 34]. 440 out of 2622 participants progressed to mechanical ventilation in the tocilizumab group compared to 536 out of 2526 participants in the other group. Statistical significance was observed with RR of 0.80 and $95 \%$ CI $0.71-0.89$ with a $p$ value $<0.0001$ (Fig. 3B). 
Fig. 1 PRISMA flow chart outlining literature search
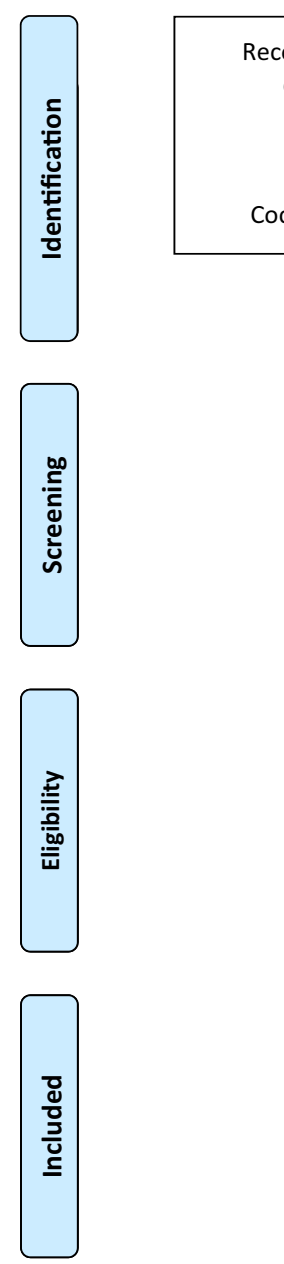
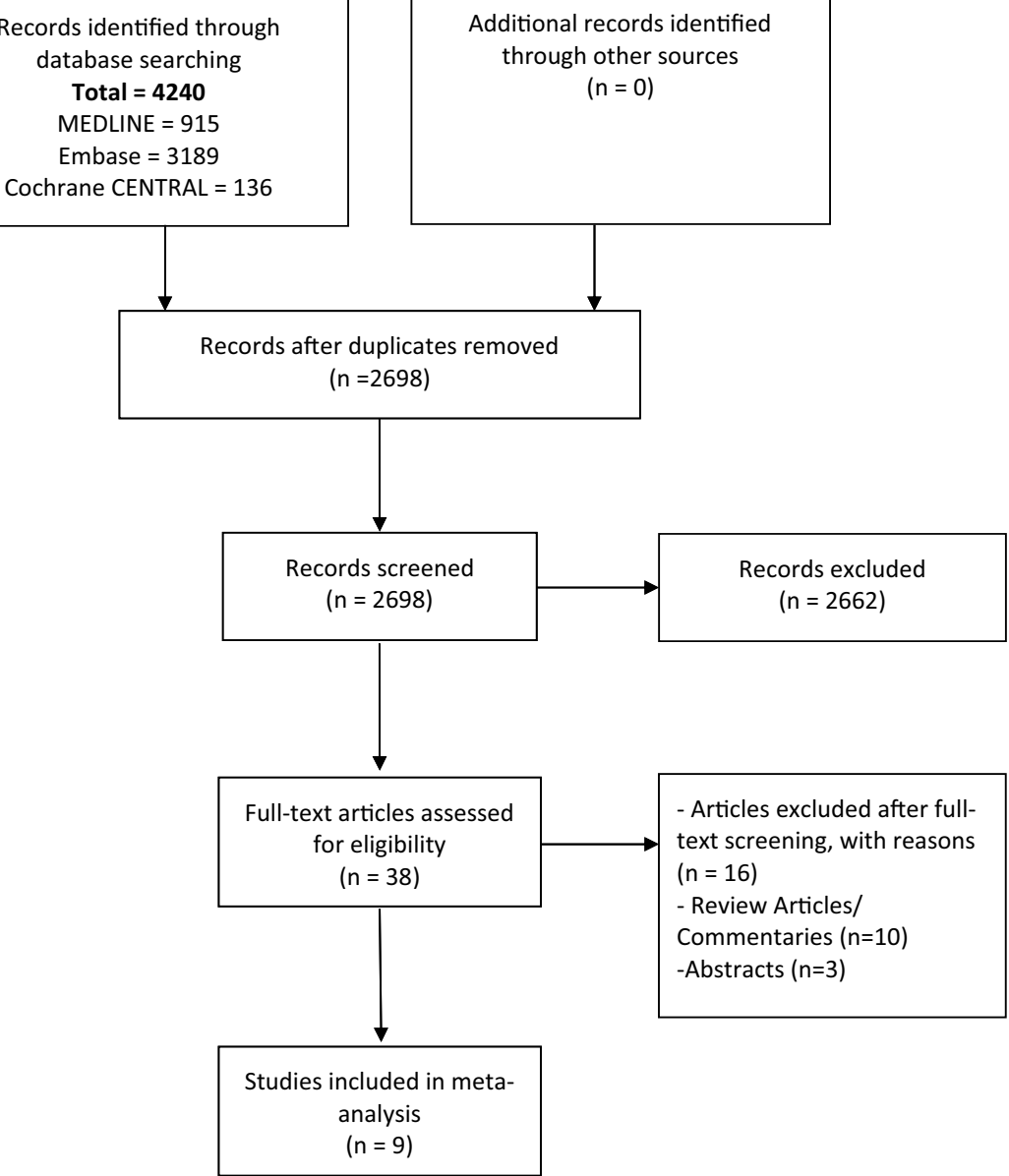

size on all-cause mortality. Five trials reported all-cause mortality in patients with severe or critical disease (on non-invasive or invasive ventilation). A total of 802 deaths out of 2825 participants were reported in the tocilizumab arm compared to 920 deaths out of 2792 participants in the standard care or placebo arm (RR 0.89, 95\% CI 0.75-1.04, $p=0.14)[25,29,31,33,34]$. In patients with mild or moderate disease, 44 deaths out of 533 participants were reported in the tocilizumab arm compared to 23 deaths out of 340 participants in the standard care or placebo arm (RR 1.20, 95\% CI 0.73-1.96, $p=0.47$ ) [24, 26-28] (Supplementary figure 1).

Three trials reported all-cause mortality in patients who received tocilizumab and dexamethasone. In patients that received dexamethasone along with tocilizumab, a total of 745 deaths out of 2624 participants were reported in the tocilizumab arm compared to 882 deaths out of 2624 participants in the standard care or placebo arm. Pooled analysis showed a significant reduction in all-cause mortality with tocilizumab therapy than standard therapy or placebo (RR 0.87, 95\% CI 0.80-0.95, $p=0.0009$ ) [26, 31, 33] (Supplementary figure 2 ). 


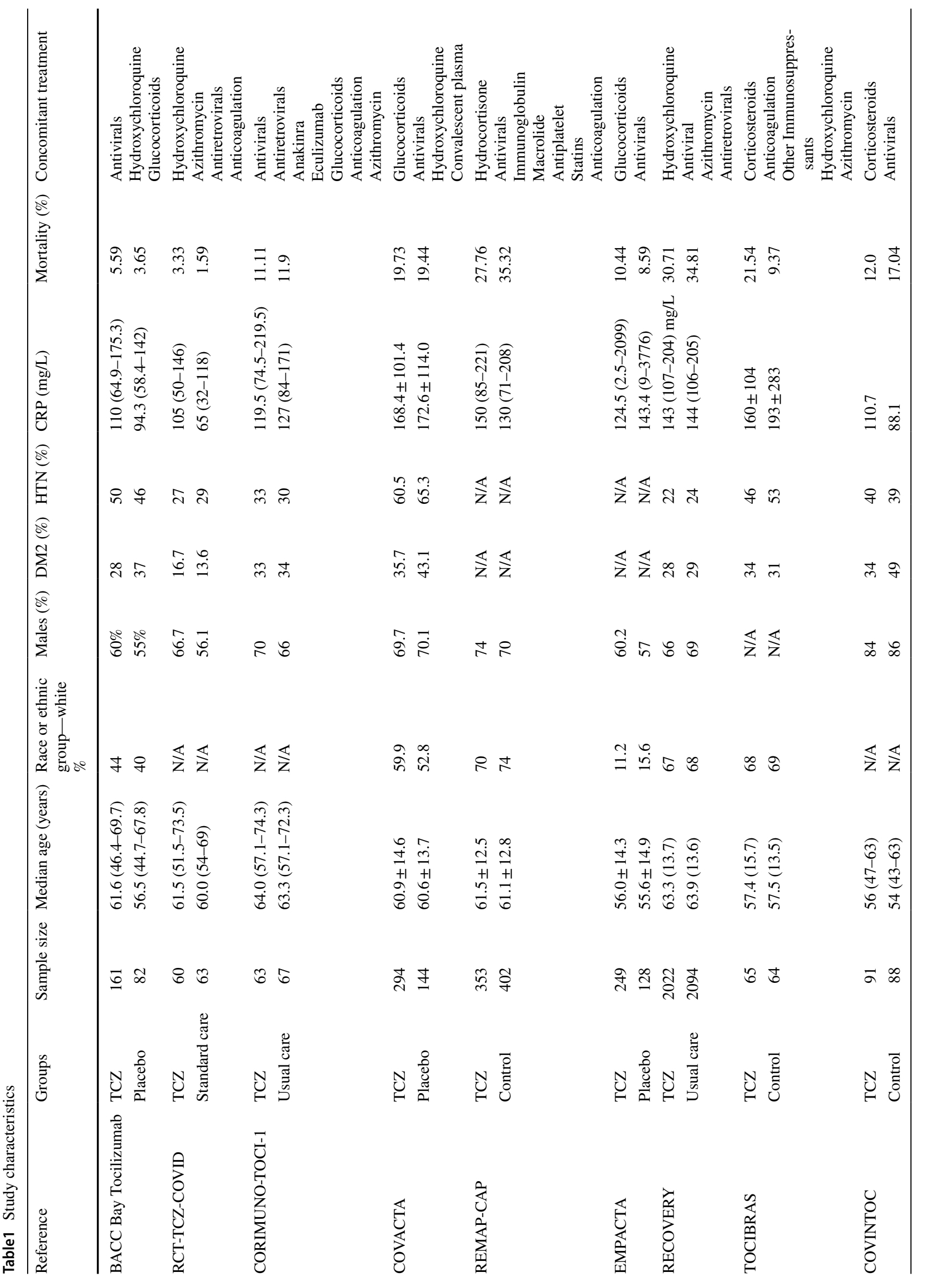




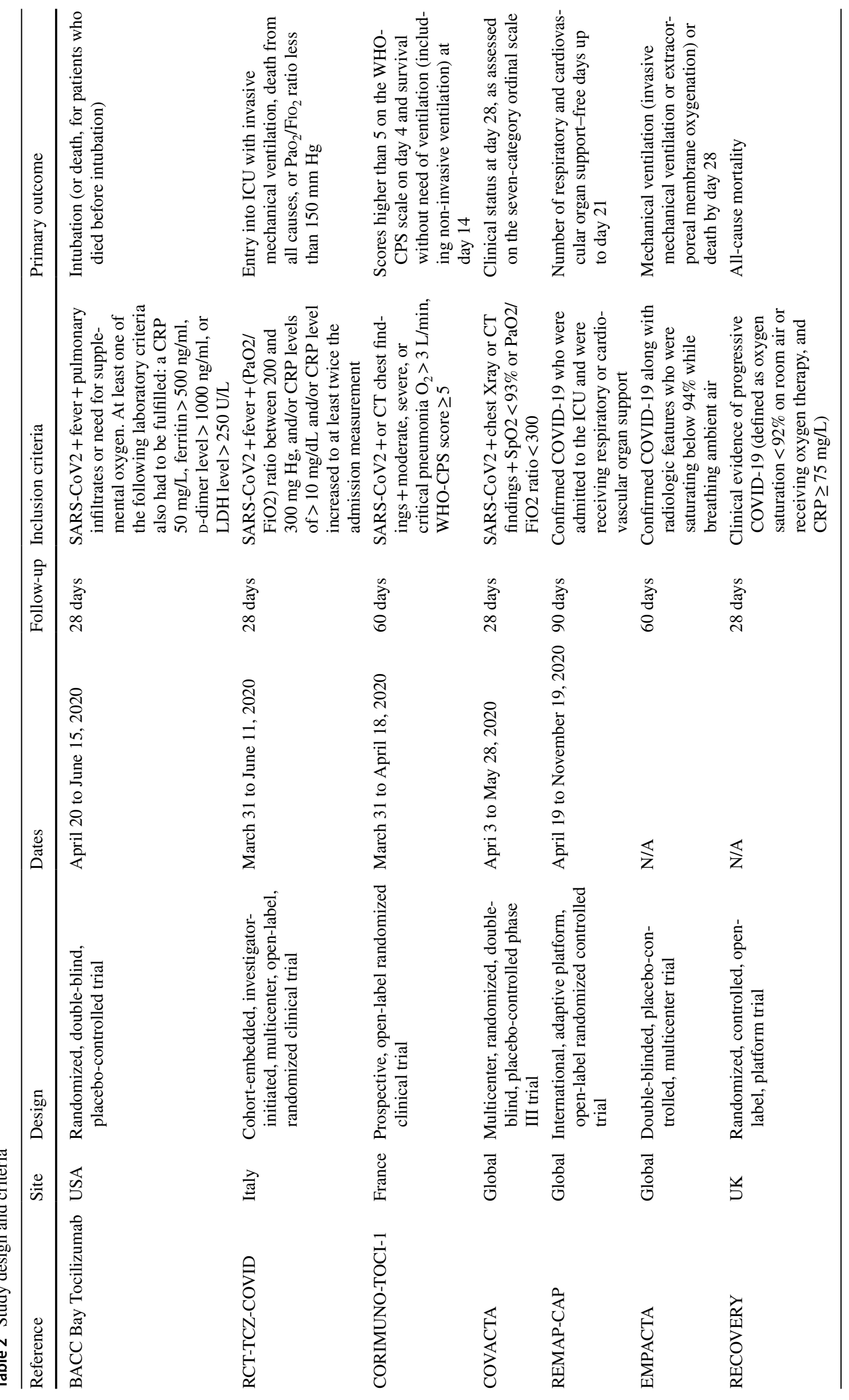




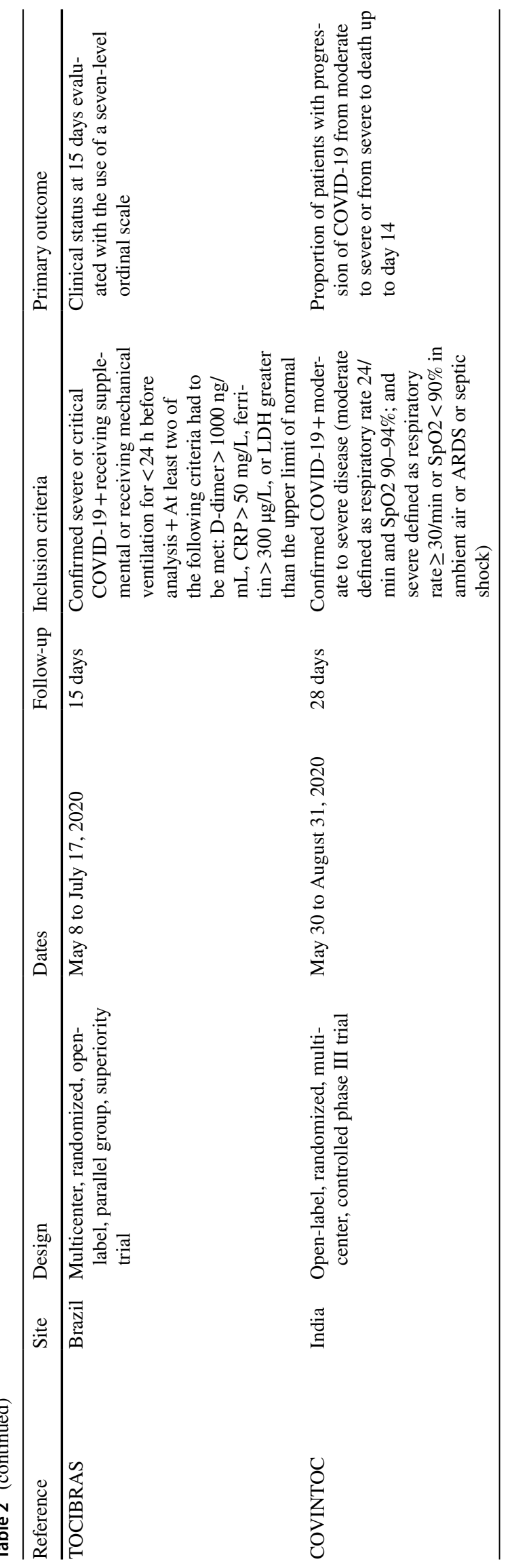

A

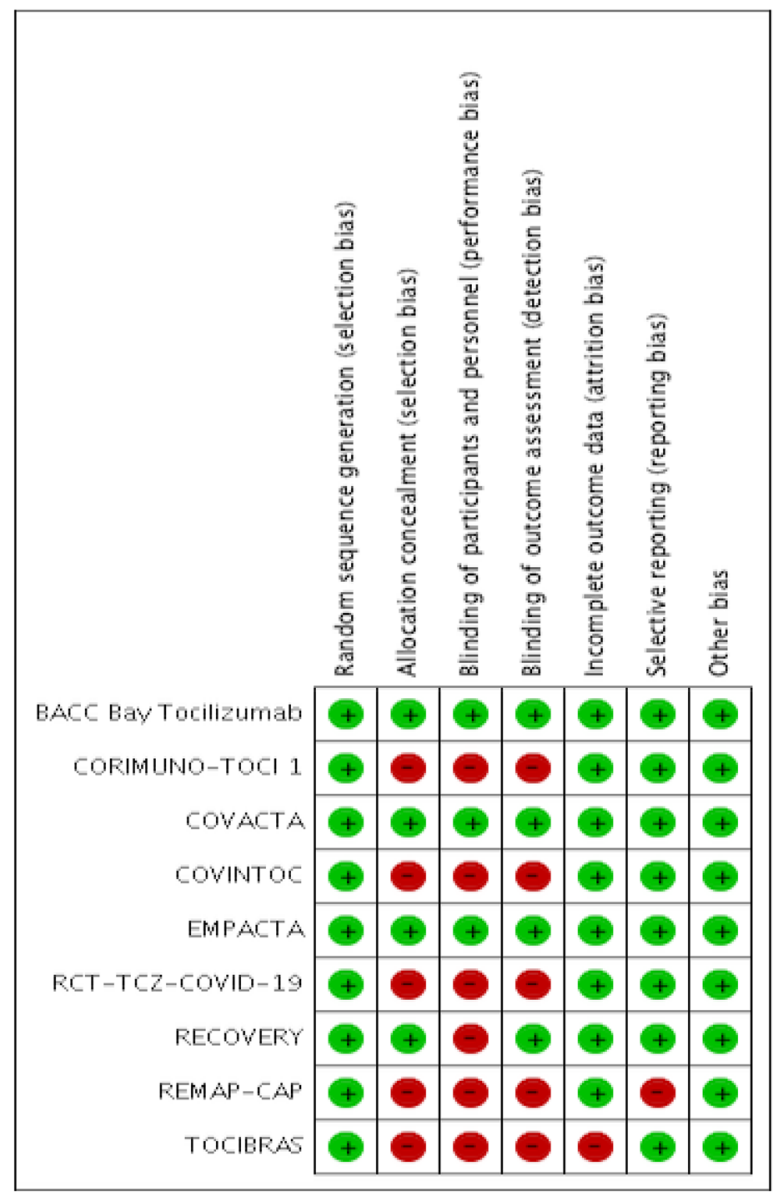

B

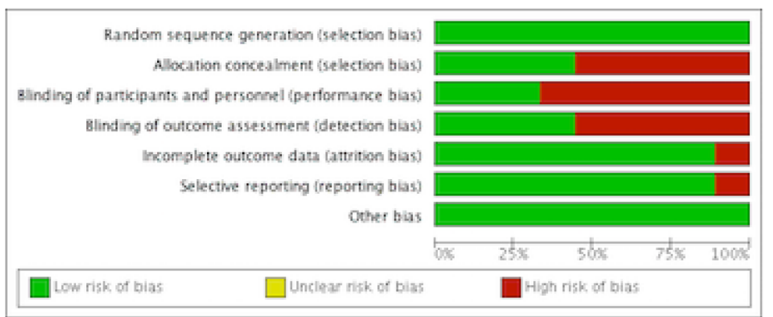

Fig. 2 Risk of bias assessment of trials included in the study

Four RCTs enrolled more than 100 patients in each arm. In these trials, there was a statistically significant reduction in all-cause mortality in patients who received tocilizumab compared to standard care or placebo (RR 0.88, 95\% CI 0.81-0.95, $p=0.001)[25,26,31,33]$. However, in RCTs with less than 100 patients in each arm, all-cause mortality outcomes were not statistically significant (RR $1.16,95 \%$ CI $0.68-1.98$, $p=0.59$ ) $[24,27-29,34]$ (Supplementary figure 3). 


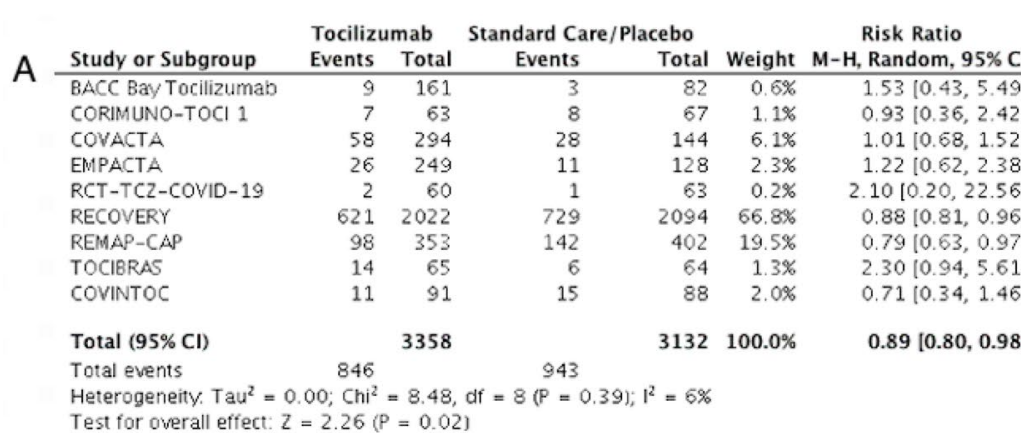

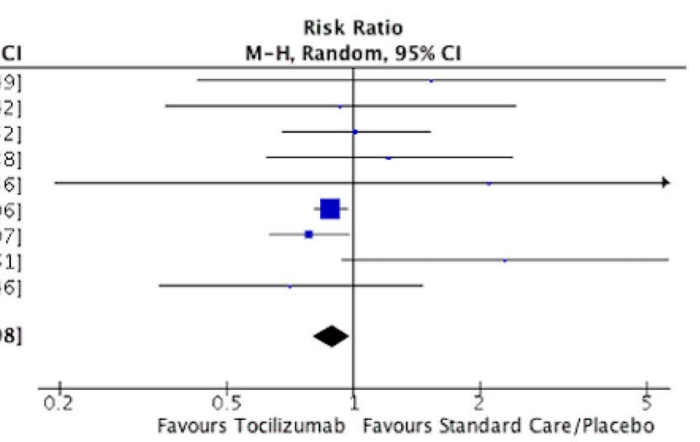

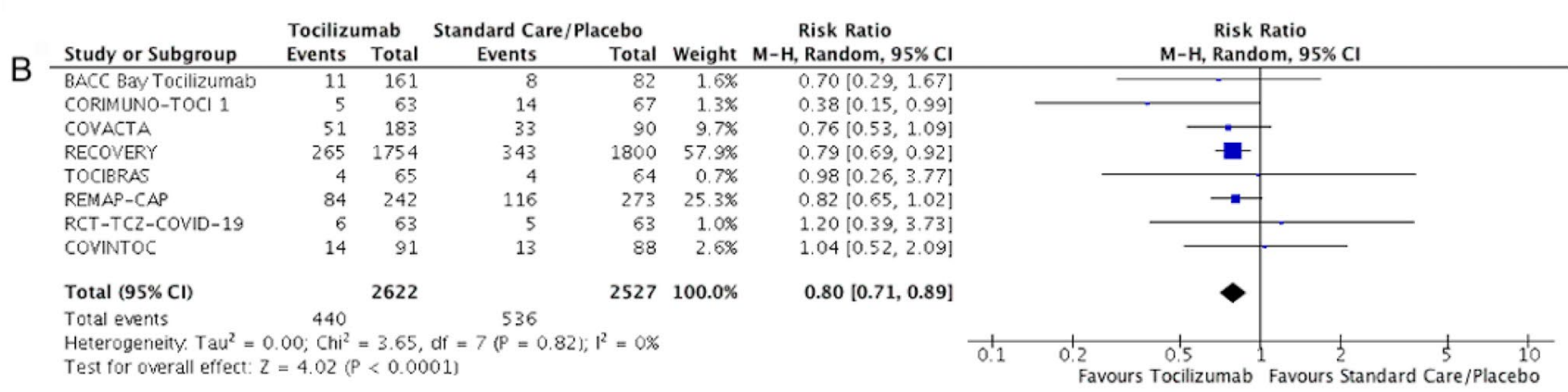

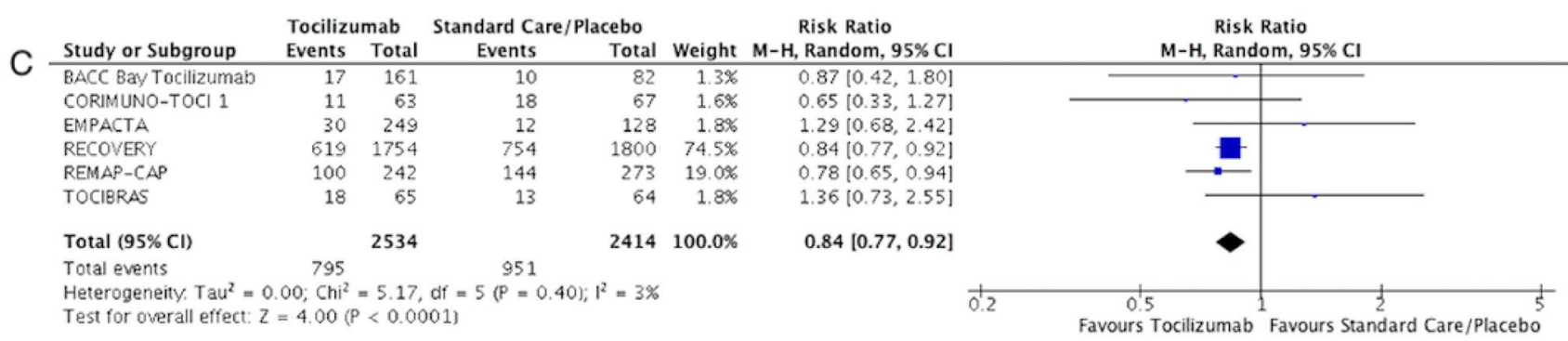

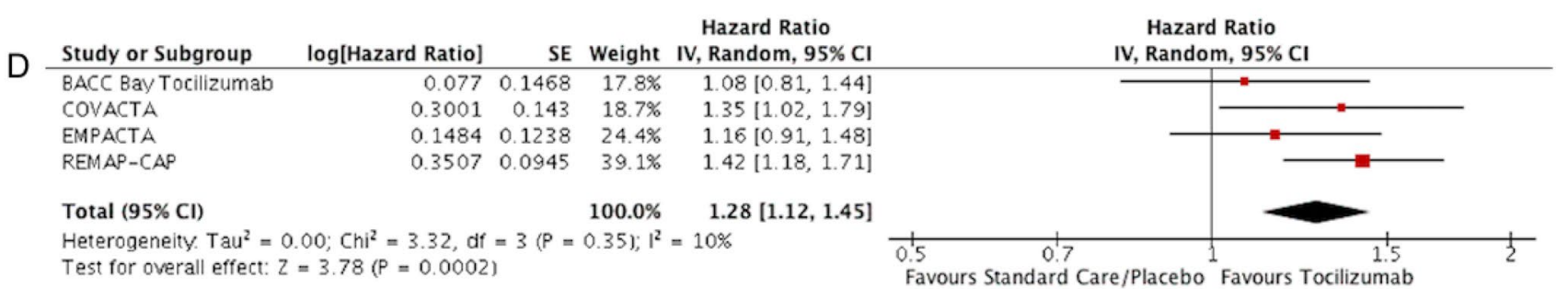

Fig. 3 Forest plots for primary and secondary outcomes. A Mortality outcome. B Progression to mechanical ventilation. C Progression to mechanical ventilation or death. D Time to discharge

\section{Discussion}

This meta-analysis provides a comprehensive aggregate analysis of the available randomized trials to date on the efficacy and safety of tocilizumab therapy in hospitalized patients with COVID-19 Infection. The results of this study favored mortality benefit with tocilizumab treatment in hypoxemic COVID-19 patients, even though they were not statistically significant. Tocilizumab therapy was also associated with reduced progression to mechanical ventilation and early hospital discharge or readiness to discharge than standard therapy or placebo in hospitalized COVID-19 patients.

Recent Infectious Diseases Society of America (IDSA) guidelines conditionally suggest the use of tocilizumab in addition to standard of care rather than standard care alone among hospitalized patients with progressive severe or critical COVID-19 [35]. The National Institutes of Health (NIH) guidelines also recommend the use of tocilizumab along with dexamethasone in hospitalized patients who have been admitted to the intensive care unit within the prior $24 \mathrm{~h}$ and who require invasive mechanical ventilation, non-invasive 
mechanical ventilation, or high-flow nasal cannula oxygen (>0.4 FiO2/30 L/min of oxygen flow). They also recommend the use of tocilizumab in hospitalized patients with rapidly increasing oxygen needs and with significantly increased markers of inflammation [36]. Our study findings support these guidelines, especially in patients with severe or critical disease in whom tocilizumab use favored improved mortality.

The hyperinflammation phase in COVID-19 involves several cytokines and chemokines. However, tocilizumab only inhibits one cytokine, IL-6. In the RCT-TCZ-COVID study, only $4 \%$ of the patient population received steroids. Tocilizumab use did not reduce the risk of clinical worsening in the study population [27]. Also, in the BACC Bay Tocilizumab study, only $10 \%$ of the study population received glucocorticoids [24]. There was no significant effect on the risk of intubation or death, on disease worsening, on time to discontinuation of supplemental oxygen. With concomitant dexamethasone administration, there appears to be a synergistic or additive effect on numerous inflammatory pathways. In the EMPACTA trial, 55.4\% of the patients in the tocilizumab group and $67.2 \%$ of those in the placebo group received concomitant dexamethasone [26]. Patients who received tocilizumab were less likely than those who received placebo to undergo mechanical ventilation or die by day 28. In the REMAP-CAP trial, steroid use increased to $88 \%$, and tocilizumab was found to improve mortality and time to clinical improvement [31]. In the RECOVERY trial, $82 \%$ of the patients received dexamethasone and tocilizumab resulted in a $6 \%$ reduction in mortality when combined with dexamethasone but had no impact on mortality given alone [33].

The severity of illness seems to play an important role in determining the benefit of tocilizumab in this population. In the RCT-TCZ-COVID study, only patients with $\mathrm{PaO} 2 /$ $\mathrm{FiO} 2$ ratios between 200 and $300 \mathrm{~mm}$ were included [27]. In the BACC Bay tocilizumab study, patients requiring $>10 \mathrm{~L} /$ min oxygen were excluded [24]. In both studies, primary outcomes were not met. In the CORIMUNO-TOCI-1 trial, patients with a WHO-CPS score of 5 with $\mathrm{O} 2$ levels of $3 \mathrm{~L} /$ min or higher but without non-invasive ventilation (NIV) or mechanical ventilation (MV) were enrolled. Survival without invasive or non-invasive mechanical ventilation by day 14 was met, but mortality at day 28 was not different between the groups. Effects of tocilizumab may have also been diminished due to greater steroid use in the control group [28]. In the COVACTA trial, $38 \%$ of the patients were mechanically ventilated. There was no significant difference between the tocilizumab and placebo groups concerning clinical status or mortality at day 28 , although the time to hospital discharge was shorter with tocilizumab (HR 1.35; 95\% CI 1.02-1.79). Besides, patients initially located outside the ICU were less likely to be transferred to the ICU if treated with tocilizumab [25]. In the REMAP-CAP trial, the greatest benefit was seen in patients admitted to the ICU for organ support (e.g., high-flow nasal cannula or ventilation) [31].

Our meta-analysis has certain limitations. Firstly, the number of patients in the RECOVERY trial was much higher than other RCTs (study weight 66.8\%) [33]. Secondly, there were differences in enrollment criteria, the time at which anti-interleukin- 6 therapy was initiated, the primary outcome, and background care. Thirdly, five studies enrolled less than 100 patients in each arm of the trial. Due to the small sample size, they may not have been powered adequately to detect a statistical difference in outcomes. Lastly, six of the included studies had an open-label design, implying high risk of performance and selection bias due to lack of blinding of participants and personnel to intervention, limiting our ability to interpret the results.

In conclusion, our meta-analysis suggests tocilizumab, when used along with dexamethasone, could be an effective therapeutic option with promising evidence on reduced mortality, progression to mechanical ventilation, and early discharge from hospital. Variations in inflammatory cascade pathophysiology make the timing of initiating treatment with tocilizumab crucial. Future studies could assess the timing of intervention based on associated comorbidities and inflammatory markers, the economic benefits of tocilizumab and other IL-6 inhibitors in patient outcomes, and critical healthcare resource usage.

Supplementary Information The online version contains supplementary material available at https://doi.org/10.1007/s00408-021-00451-9.

Author Contributions MSK involved in conceptualization, methodology, and software. VS participated in data curation and writingoriginal draft preparation. CB did visualization, investigation, and validation. KD-A and EM did supervision. AF and AL performed writing - reviewing and editing.

Funding None.

\section{Declarations}

Conflict of interest On behalf of all authors, the corresponding author states that there is no conflict of interest.

\section{References}

1. Wu Z, McGoogan JM (2020) Characteristics of and important lessons from the coronavirus disease 2019 (COVID-19) outbreak in China: summary of a report of 72314 cases from the Chinese Center for Disease Control and Prevention. JAMA 323(13):12391242. https://doi.org/10.1001/jama.2020.2648

2. Siddiqi HK, Mehra MR (2020) COVID-19 illness in native and immunosuppressed states: A clinical-therapeutic staging proposal. 
J Heart Lung Transplant. https://doi.org/10.1016/j.healun.2020.03. 012

3. Wang D, Hu B, Hu C et al (2020) Clinical characteristics of 138 hospitalized patients with 2019 novel coronavirus-infected pneumonia in Wuhan China. JAMA. https://doi.org/10.1001/jama.2020.1585

4. Zhou F, Yu T, Du R et al (2020) Clinical course and risk factors for mortality of adult inpatients with COVID-19 in Wuhan, China: a retrospective cohort study. The Lancet 395(10229):1054-1062

5. Kermali $\mathrm{M}$ et al (2020) The role of biomarkers in diagnosis of COVID-19—a systematic review. Life Sci. https://doi.org/10.1016/j. lfs. 2020.117788

6. Costela-Ruiz VJ et al (2020) SARS-CoV-2 infection: the role of cytokines in COVID-19 disease. Cytokine Growth Factor Rev 54:62-75. https://doi.org/10.1016/j.cytogfr.2020.06.001

7. Pons S, Fodil S, Azoulay E, Zafrani L (2020) The vascular endothelium: the cornerstone of organ dysfunction in severe SARS-CoV-2 infection. Crit Care 24:353-353

8. Mehta P, McAuley DF, Brown M et al (2020) COVID-19: consider cytokine storm syndromes and immunosuppression. Lancet 395:1033-1034

9. Giamarellos-Bourboulis EJ, Netea MG, Rovina N et al (2020) Complex immune dysregulation in COVID-19 patients with severe respiratory failure. Cell Host Microbe 27(6):992-1000.e3

10. Chen X, Zhao B, Qu Y et al (2020) Detectable serum SARS-CoV-2 viral load (RNAaemia) is closely correlated with drastically elevated interleukin 6 (IL-6) level in critically ill COVID-19 patients. Clin Infect Dis. https://doi.org/10.1093/cid/ciaa449

11. Lin A, He Z-B, Zhang S, Zhang J-G, Zhang X, Yan W-H (2020) Early risk factors for the duration of SARS-CoV-2 viral positivity in COVID-19 patients. Clin Infect Dis 71(16):2061-2065

12. Herold T, Jurinovic V, Arnreich C et al (2020) Elevated levels of IL-6 and CRP predict the need for mechanical ventilation in COVID-19. J Allergy Clin Immunol 146(1):128-136.e4

13. Del Valle DM, Kim-Schulze S, Huang HH et al (2020) An inflammatory cytokine signature predicts COVID-19 severity and survival. Nat Med 26(10):1636-1643

14. Coomes EA, Haghbayan H (2020) Interleukin-6 in COVID-19: a systematic review and meta-analysis. Rev Med Virol 30(6):1-9

15. RECOVERY Collaborative Group, Horby P, Lim WS, Emberson JR et al (2021) Dexamethasone in hospitalized patients with Covid19. N Engl J Med 384(8):693-704. https://doi.org/10.1056/NEJMo a2021436

16. Tomazini BM, Maia IS, Cavalcanti AB et al (2020) Effect of dexamethasone on days alive and ventilator-free in patients with moderate or severe acute respiratory distress syndrome and COVID-19: the CoDEX randomized clinical trial. JAMA 324(13):1307-1316. https://doi.org/10.1001/jama.2020.17021

17. Manfredi A, Cassone G, Furini F et al (2020) Tocilizumab therapy in rheumatoid arthritis with interstitial lung disease: a multicenter retrospective study. Intern Med J 50(9):1085-1090. https://doi.org/ 10.1111/imj. 14670

18. Schirmer M, Muratore F, Salvarani C (2018) Tocilizumab for the treatment of giant cell arteritis. Expert Rev Clin Immunol 14(5):339-349. https://doi.org/10.1080/1744666X.2018.1468251

19. Le RQ, Li L, Yuan W et al (2018) FDA approval summary: tocilizumab for treatment of chimeric antigen receptor $\mathrm{T}$ cell-induced severe or life-threatening cytokine release syndrome. Oncologist 23(8):943-947. https://doi.org/10.1634/theoncologist.2018-0028

20. Nishimoto N, Kishimoto T (2008) Humanized antihuman IL-6 receptor antibody, tocilizumab. Handb Exp Pharmacol 181:151160. https://doi.org/10.1007/978-3-540-73259-4_7

21. Pairo-Castineira E, Clohisey S, Klaric L, GenOMICC Investigators, ISARIC4C Investigators, COVID-19 Human Genetics Initiative, 23andMe Investigators, BRACOVID Insvestigators, Gen-COVID Investigators, Shen X, Ponting CP, Fawkes A, Tenesa A, Caulfield M, Scott R, Rowan K, Murphy L, Openshaw PJM, Semple MG, Law
A, Vitart V, Wilson JF, Baillie JK et al (2021) Genetic mechanisms of critical illness in COVID-19. Nature 591(7848):92-98. https:// doi.org/10.1038/s41586-020-03065-y

22. Xu X, Han M, Li T et al (2020) Effective treatment of severe COVID-19 patients with tocilizumab. Proc Natl Acad Sci USA 117:10970-10975

23. Khan FA, Stewart I, Fabbri L et al (2021) Systematic review and meta-analysis of anakinra, sarilumab, siltuximab and tocilizumab for COVID-19. Thorax. https://doi.org/10.1136/thoraxjnl-2020-215266

24. Stone JH, Frigault MJ, Serling-Boyd NJ, BACC Bay Tocilizumab Trial Investigators et al (2020) Efficacy of tocilizumab in patients hospitalized with Covid-19. N Engl J Med 383(24):2333-2344. https://doi.org/10.1056/NEJMoa2028836

25. Rosas IO, Bräu N, Waters M, Go RC, Hunter BD, Bhagani S et al (2021) Tocilizumab in hospitalized patients with severe Covid-19 pneumonia. N Engl J Med. https://doi.org/10.1056/NEJMoa2028 700

26. Salama C, Han J, Yau L et al (2021) Tocilizumab in patients hospitalized with Covid-19 pneumonia. N Engl J Med 384(1):20-30. https://doi.org/10.1056/NEJMoa2030340

27. Salvarani C, Dolci G, Massari M, RCT-TCZ-COVID-19 Study Group et al (2021) Effect of tocilizumab vs standard care on clinical worsening in patients hospitalized with COVID-19 pneumonia: a randomized clinical trial. JAMA Intern Med 181(1):24-31. https:// doi.org/10.1001/jamainternmed.2020.6615

28. Hermine O, Mariette X, Tharaux PL, CORIMUNO-19 Collaborative Group et al (2021) Effect of tocilizumab vs usual care in adults hospitalized with COVID-19 and moderate or severe pneumonia: a randomized clinical trial. JAMA Intern Med 181(1):32-40. https:// doi.org/10.1001/jamainternmed.2020.6820

29. Veiga VC, Prats JAGG, Farias DLC, Coalition covid-19 Brazil VI Investigators et al (2021) Effect of tocilizumab on clinical outcomes at 15 days in patients with severe or critical coronavirus disease 2019: randomised controlled trial. BMJ. https://doi.org/10.1136/ bmj.n84

30. Moher D, Liberati A, Tetzlaff J, Altman DG, Group P (2009) Preferred reporting items for systematic reviews and meta-analyses: the PRISMA statement. BMJ 339:b2535

31. REMAP-CAP Investigators, Gordon AC, Mouncey PR, Al-Beidh F et al (2021) Interleukin-6 receptor antagonists in critically Ill patients with Covid-19. N Engl J Med. https://doi.org/10.1056/NEJMoa2100 433

32. Higgins JP, Altman DG, Gotzsche PC et al (2011) The Cochrane Collaboration's tool for assessing risk of bias in randomised trials. BMJ. 18:343

33. RECOVERY Collaborative Group (2021) Tocilizumab in patients admitted to hospital with COVID-19 (RECOVERY): a randomised, controlled, open-label, platform trial. Lancet 397(10285):1637-1645

34. Soin AS, Kumar K, Choudhary NS et al (2021) Tocilizumab plus standard care versus standard care in patients in India with moderate to severe COVID-19-associated cytokine release syndrome (COVINTOC): an open-label, multicentre, randomised, controlled, phase 3 trial. Lancet Respir Med. https://doi.org/10.1016/S2213-2600(21) 00081-3

35. Infectious Diseases Society of America (IDSA) guidelines on the Treatment and Management of Patients with COVID-19. https:// www.idsociety.org/practice-guideline/covid-19-guideline-treatmentand-management/. Accessed 5 Mar 2021

36. National Institutes of Health (NIH) COVID-19 Treatment Guidelines. The COVID-19 Treatment Guidelines panel's statement on the use of tocilizumab for the treatment of COVID-19 https://www. covid19treatmentguidelines.nih.gov/statement-on-tocilizumab/. Accessed 5 Mar 2021

Publisher's Note Springer Nature remains neutral with regard to jurisdictional claims in published maps and institutional affiliations. 